\title{
Ultrametric \\ Banach Algebras
}


This page is intentionally left blank 


\section{Ultrametric \\ Banach Algebras}

Alain Escassut

Université Blaise Pascal, France 


\section{Published by}

World Scientific Publishing Co. Pte. Ltd.

5 Toh Tuck Link, Singapore 596224

USA office: Suite 202, 1060 Main Street, River Edge, NJ 07661

UK office: 57 Shelton Street, Covent Garden, London WC2H 9HE

\section{British Library Cataloguing-in-Publication Data}

A catalogue record for this book is available from the British Library.

\section{ULTRAMETRIC BANACH ALGEBRAS}

Copyright $\odot 2003$ by World Scientific Publishing Co. Pte. Ltd.

All rights reserved. This book, or parts thereof, may not be reproduced in any form or by any means, electronic or mechanical, including photocopying, recording or any information storage and retrieval system now known or to be invented, without written permission from the Publisher.

For photocopying of material in this volume, please pay a copying fee through the Copyright Clearance Center, Inc., 222 Rosewood Drive, Danvers, MA 01923, USA. In this case permission to photocopy is not required from the publisher.

ISBN $981-238-194-5$

This book is printed on acid-free paper.

Printed in Singapore by Mainland Press 


\section{Contents}

Introduction $\quad$ ix

1. Basic Properties in Commutative Algebra 1

2. Tree Structure 9

3. Ultrametric Absolute Values 13

4. L-Productal Vector Spaces 17

5. Multiplicative Semi-Norms and Shilov Boundary 21

6. Spectral Semi-Norm 31

$\begin{array}{ll}\text { 7. Hensel Lemma } & 37\end{array}$

8. Infraconnected Sets 45

9. Monotonous Filters 51

10. Circular Filters 57

11. Tree Structure and Metric on Circular Filters 65

12. Rational Functions and Algebras $R(D) \quad 71$

13. Simple Convergence on $\operatorname{Mult}(K[x]) \quad 75$

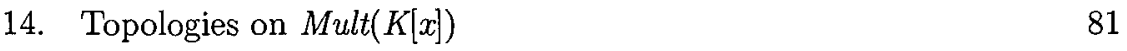

15. Spectral Properties and Gelfand Transforms 87 
16. Analytic Elements 93

17. Holomorphic Properties on Infraconnected Sets 101

18. T-Filters and $T$-Sequences 105

19. Applications of $T$-Filters and $T$-Sequences 113

20. Analytic Elements on Classic Partitions 119

21. Holomorphic Properties on Partitions 123

22. Shilov Boundary for Algebras $H(D, \mathcal{O}) \quad 127$

23. Holomorphic Functional Calculus 135

24. Uniform $K$-Banach Algebras and Properties ( $s$ ) and $(q) \quad 143$

25. Properties $(o)$ and $(q)$ in Uniform Banach $K$-Algebras $\quad 149$

26. Properties $(o)$ and $(q)$ and Strongly Valued Fields 161

27. Multbijective Banach K-Algebras 167

28. Pseudo-Density of $\operatorname{Mult}_{m}(A,\|\cdot\|) \quad 171$

29. Polnorm on Algebras and Algebraic Extensions 175

30. Definition of Affinoid Algebras 181

31. Algebraic Properties of Affinoid Algebras 187

32. Jacobson Radical of Affinoid Algebras 193

$\begin{array}{ll}\text { 33. Salmon's Theorems } & 197\end{array}$

34. Separable Fields 201

35. Spectral Norm of Affinoid Algebras 209

36. Spectrum of an Element of an Affinoid Algebra 215

37. Krasner-Tate Algebras 221

38. Universal Generators in Tate Algebras 227

39. Mappings from $H(D)$ to the Tree $\operatorname{Mult}(K[x])$ 
40. Continuous Mappings on $\operatorname{Mult}(K[x]) \quad 239$

41. Examples and Counterexamples 247

41. Associated Idempotents 255

43. Krasner-Tate Algebras among Banach $K$-Algebras 259

$\begin{array}{ll}\text { References } & 265\end{array}$

$\begin{array}{ll}\text { Definitions Index } & 269\end{array}$

$\begin{array}{ll}\text { Notation Index } & 273\end{array}$ 
This page is intentionally left blank 


\section{Introduction}

Thanks to Gelfand's theory, semi-simple commutative complex Banach algebras may be seen as algebras of functions on a compact set whose points characterize all maximal ideals, which all are of codimension 1. Moreover, it is known that all multiplicative semi-norms, continuous with respect to the $\mathbb{C}$-algebra norm, are of the form $|\chi|$, with $\chi$ a $\mathbb{C}$-algebra homomorphism. Given an element $x \in A$, it is possible to define a holomorphic mapping from its spectrum to the algebra: this is called the holomorphic functional calculus.

Now, consider an algebraically closed complete ultrametric field $K$ and a commutative ultrametric Banach $K$-algebra $A$ with unity. Such an algebra may have maximal ideals of infinite codimension. Anyway, it is not possible to make it isomorphic to an algebra of functions (with values in $K$ ) defined on a compact set. Trying to construct a (basic) spectral theory on $A$ is then much more difficult than in Archimedean analysis. Following basic works by Monna and T.A. Springer, B. Guennebaud saw the importance of continuous multiplicative semi-norms on such an ultrametric Banach $K$ algebras [34]. More recently, V. Berkovich also considered the theory of multiplicative semi-norms and enjoyed it to construct an improved generalization of Tate's theory $[1,49]$. Here we particularly make a kind of Gelfand transform which associates to any element $f$ of the algebra $A$ a function $f^{*}$ defined on the set of multiplicative continuous semi-norms of $A$, with values in the set $M u l t(K[x])$ of multiplicative semi-norms of $K[x]$, also called the one dimensional Berkovich affine space. Thus, among continuous multiplicative semi-norms of $A$, we find those whose kernel is a maximal ideal 
of codimension 1 , and then the restriction of $f^{*}$ to them appears similar to the classical definition in Archimedean analysis.

Our way to examine semi-norms consists of characterizing them by circular filters $[32,30]$. Moreover, the set of circular filters has a tree structure which is easily described: every pair admits a supremum, and if two elements $b, c$ are bigger than another $a$, then $b$ and $c$ are comparable. The set is provided with two topologies: the simple convergence, induced by the topology on continuous multiplicative semi-norms on $K[x]$ (for which it is locally compact and every compact is sequentially compact), and a metric topology whose definition is closely linked to the tree structure. This metric defines a topology which is strictly stronger than the simple convergence: the set of circular filers is not locally compact for the metric topology, but it is complete. Following a study by K. Boussaf, an analytic element or a meromorphic function roughly transforms a circular filter into another one. Since the field $K$ appears as a subset of the set of circular filters, such mappings are continuous for both topologies on the set of circular filters. A meromorphic function is increasing with respect to the order on the set of circular filters if and only if it is an entire function. And when two functions are close enough, then give the same image of a circular filter. On the other hand, when the field $K$ is topologically separable i.e. has a countable dense subset, it is possible to show that the simple convergence is metrizable and then we construct another metric defining the simple convergence [39].

In a Banach $K$-algebra $A$ there does exist a spectral semi-norm, defined as $\|x\|_{s i}=\lim _{n \rightarrow \infty}\left\|x^{n}\right\|^{\frac{1}{n}}$, and it is equal to the supremum of all continuous multiplicative semi-norms. It is easy to construct examples where this spectral norm is strictly superior to the supremum of multiplicative seminorms whose kernel is a maximal ideal of codimension 1, which gives an idea of the problems we risk encountering. Meanwhile, every maximal ideal is the kernel of (at least) one continuous multiplicative semi-norm (this is not the case of certain closed prime ideals [7]). On the other hand, a Shilov boundary exists for continuous multiplicative semi-norms, relatively to the spectral semi-norm and we construct it by following a method due to $\mathrm{B}$. Guennebaud. In the particular case of a Krasner algebra $H(D)$, the Shilov boundary consists of circular filters secant with both $D$ and $K \backslash D$ [8].

Since 1975, it has been possible to define a kind of holomorphic functional calculus in $A$ [23]: given $x \in A$, thanks to the ultrametric property of the norm, we can show that if $a, b$ are in a same hole of the spectrum of $x$, and such that $|b-a|<\frac{1}{\left\|(x-a)^{-1}\right\|}$, then $\left\|(x-a)^{-1}\right\|=\left\|(x-b)^{-1}\right\|$. 
Thanks to this property, combined with the Krasner-Mittag-Leffler Theorem, it is possible to define, on the algebra of rational functions with no poles in the spectrum of $x$, an ultrametric norm that makes continuous the natural homomorphism from this algebra into $A$. As a consequence, many problems on ultrametric Banach algebras may be studied through the set Mult $(K[x])$.

So, in a few words, we can say that the book consists of associating methods based on affinoid algebras (also called "Tate algebras") and methods based on holomorphic functional calculus involving very thin property on analytic functions in one variable. Particularly, we can consider the behaviour of multiplicative semi-norms on the algebra of rational functions with no pole in the spectrum of $x$ by using properties of analytic functions. In such considerations, $T$-filters play a central role. This $K$-subalgebra $B$ of $K(x)$ is then provided with an ultrametric norm defined with help of the relation above, and its completion $\widehat{B}$ looks like a Krasner algebra. This way, we can define a natural continuous homomorphism from $\widehat{B}$ into $A$, and therefore find in $A$ properties known in $\widehat{B}$.

The problem whether a maximal ideal of infinite codimension may be the kernel of several continuous multiplicative semi-norms had an answer by 1976 [21]: thanks to properties of $T$-filters, when the field $K$ is "weakly valued" i.e. both its residue class field and its value group are countable, then it is possible to construct an example where an infinity of continuous multiplicative semi-norms admit for kernel a same maximal ideal: for instance $\mathbb{C}_{p}$ is weakly valued. However, if at least one of these two sets is not countable, then each maximal ideal is the kernel of only one continuous multiplicative semi-norm [23].

Another problem arose: is the spectral norm equal to the supremum of all multiplicative semi-norms whose kernel is a maximal ideal? When the field $K$ is strongly valued, the answer is yes [23]. But when it is weakly valued, if there is no particular hypothesis on the norm of $A$, the answer is "no": there even exist certain ultrametric Banach $K$-algebras admitting elements in the Jacobson radical, which have a spectral norm different from 0 [23]. However, when the algebra is complete with respect to its spectral norm, the answer is yes [29]. Certain other spectral properties appear to be linked to integrity.

The set of continuous multiplicative semi-norms whose kernel is a maximal ideal is known to be dense in the whole set of all continuous multiplicative semi-norms in many simple examples, such as Krasner algebras and affinoid algebras. As a consequence of properties of the spectral norm 
described above, it is at least pseudo-dense when either the norm is the spectral one, or the field is strongly valued [29].

Affinoid algebras form a large panel of commutative ultrametric Banach $K$-algebras [49]. We recall the proofs of their algebraic and topological properties: they are noetherian Jacobson rings whose maximal ideals have codimension 1, and, in a reduced affinoid algebra, the spectral semi-norm is equivalent to the Banach algebra norm (a property which is not easily proven in characteristic $p \neq 0$ ). Moreover, topologically pure extensions are factorial [45]. By showing that the set of continuous multiplicative semi-norms whose kernel is a maximal ideal is dense in the whole set of all continuous multiplicative semi-norms [34], we have an easier proof of the Jacobson ring property.

An infraconnected subset of $K$ is called affinoid [2] (or ultracirconférencié [18]) if it has finitely many holes and if its envelope and all its holes have a diameter in the value set of $K$. More generally, a subset of $K$ is called affinoid (or ultracirconférencié) if it is a finite union of infraconnected affinoid subsets of $K$. Given an element $x$ of an affinoid algebra, $Y$. Morita showed that the spectrum of $x$ is an affinoid subset of $K$ [37]. Here we give a new and more simple proof of this property, based on elementary considerations. This is useful to characterize algebras that are both Krasner algebras and Tate algebras: they are called Krasner-Tate algebras [18]. They are characterized among Krasner algebras (as the algebras $H(D)$ when $D$ is affinoid), and among Tate algebras, as a quotient of the topologically pure extension $T_{2}=K\{T, Y\}$ by a principal ideal of a particular form. They are also characterized among ultrametric Banach algebras, by a set of properties, where each is logically independent from all others. A particular interest of Krasner-Tate algebras is that they make a very typical and basic class of affinoid algebras that one can easily represent as an algebra of functions defined in a set of $K$. Thanks to the mapping on the set of circular filters described above, we can characterize the expansions of the Gauss norm defined on $K\{T\}$ to continuous absolute values on a Krasner-Tate algebra $H(D)=K\{T\}[x]$ (this involves the Shilov boundary of $H(D)$ ).

With help of Krasner-Tate algebras, we can also find fine conditions to obtain an idempotent associated to a subset of the spectrum of an element in an ultrametric Banach algebra. Particularly, if the spectral semi-norm is equal to the upper bound of the spectrum, we can show that if $A$ has no non trivial idempotents, then the spectrum of every element is infraconnected. And particularly, this holds for a Banach algebra complete for its spectral 
norm. Several examples and counter-examples, provided with non usual ultrametric algebra norms, show the relative importance of such properties involved in theorems. They also give opportunities to test certain other claims or conjectures.

So far, books related to affinoid theory mainly ignored general ultrametric Banach algebra, and books dedicated to ultrametric functional analysis ignored affinoid algebras. This is why it seemed useful to gather both properties and methods. Many theorems proven in [30] are indispensable in the present book and are recalled with references, without proving them again.

I am very grateful to Jesus Araujo for reading my drafts, and noticing misprints, and to Bertin Diarra and Gabriel Picavet for advising me in basic algebra. 